\title{
Neutron spectrometer for fast nuclear reactors
}

\author{
M. Osipenko ${ }^{\text {a,* }}$, M. Ripani ${ }^{\text {a }}$, G. Ricco ${ }^{a}$, B. Caiffi ${ }^{\text {b }}$, F. Pompili ${ }^{c}$, M. Pillon $^{c}$, M. Angelone ${ }^{c}$, \\ G. Verona-Rinati ${ }^{\text {d, R. } \text { Cardarelli }^{e}, \text { G. Mila }}{ }^{\mathrm{f}}$, S. Argiro $^{\mathrm{f}}$ \\ a INFN, sezione di Genova, 16146 Genova, Italy \\ b Dipartimento di Fisica dell'Università di Genova, 16146 Genova, Italy \\ ${ }^{\mathrm{c}}$ ENEA, Frascati 00044, Italy \\ ${ }^{\mathrm{d}}$ Università di Tor Vergata, Rome 00133, Italy \\ e INFN sezione di Roma II, 00133 Italy \\ ${ }^{\mathrm{f}}$ Università di Torino and INFN, Turin 10125, Italy
}

\section{A R T I C L E I N F O}

\section{Article history:}

Received 8 June 2015

Received in revised form

24 July 2015

Accepted 24 July 2015

Available online 1 August 2015

Keywords:

Neutron spectrometer

Fast reactor

Diamond detector

\begin{abstract}
A B S T R A C T
In this paper we describe the development and first tests of a neutron spectrometer designed for high flux environments, such as the ones found in fast nuclear reactors. The spectrometer is based on the conversion of neutrons impinging on ${ }^{6} \mathrm{Li}$ into $\alpha$ and $t$ whose total energy comprises the initial neutron energy and the reaction $Q$-value. The ${ }^{6} \mathrm{LiF}$ layer is sandwiched between two CVD diamond detectors, which measure the two reaction products in coincidence. The spectrometer was calibrated at two neutron energies in well known thermal and $3 \mathrm{MeV}$ neutron fluxes. The measured neutron detection efficiency varies from $4.2 \times 10^{-4}$ to $3.5 \times 10^{-8}$ for thermal and $3 \mathrm{MeV}$ neutrons, respectively. These values are in agreement with Geant 4 simulations and close to simple estimates based on the knowledge of the ${ }^{6} \mathrm{Li}(\mathrm{n}, \alpha) t$ cross-section. The energy resolution of the spectrometer was found to be better than $100 \mathrm{keV}$ when using 2.5 or $5 \mathrm{~m}$ cables between the detector and the preamplifiers.
\end{abstract}

(c) 2015 Elsevier B.V. All rights reserved.

\section{Introduction}

The standard nuclear reactor on-line diagnosis is performed with fission chambers. Solid state detectors have a number of features which distinguish them from gas filled counters. In particular, the much faster carrier drift velocity allows for a rapid charge collection and therefore high counting rate. The high density of the active volume permits spectroscopic measurements with compact detectors which do not alter local conditions. The sensitivity to $\gamma \mathrm{s}$ is also lower for solid state detectors because of smaller size and in particular for low-Z detector materials (e.g. for diamond). However, a limitation in the application of solid state detectors to nuclear diagnostics stems from the fact that large non-ionizing energy deposition damages the crystalline structure and alters the properties of the detector. This leads to a reduction of the charge collection efficiency and, in some semiconductors, to an increase of the leakage current. Silicon detectors are strongly affected by this type of damage and cannot be used in high radiation environment. Thanks to the higher displacement energy $(43 \mathrm{eV})$ and the lower $Z$ value, diamond detectors feature up to an order of magnitude larger resistance to non-ionizing doses [1].

\footnotetext{
* Corresponding author.

E-mail address: osipenko@ge.infn.it (M. Osipenko).
}

Also the diamond shows no leakage current increase after irradiation at room temperature [2]. Therefore, several studies are available in the literature that present their application as diagnostic tools in nuclear facilities.

In addition to radiation hardness, diamond detectors also exhibit a low intrinsic noise at high temperatures thanks to their large bandgap of $5.5 \mathrm{eV}$. This proposes the diamond detectors for usage in fission reactors where the operating temperature may reach $700^{\circ}$ and near fusion plasma chambers, although necessary studies are still on-going. However, the presence of impurities in the diamond crystal creates levels inside the bandgap, thereby increasing the trapping of charge carriers and therefore the charge collection efficiency. High impurity density limited the usage of natural diamonds as radiation detectors. The advent of the Chemical Vapour Deposition (CVD) diamond growing technique enhanced the quality of diamond detectors and allowed practical applications.

Nuclear reactors in which the neutron spectra are dominated by energies well above thermal energies are called fast reactors. These include reactors featuring heavy or low density moderators. A more energetic neutron spectrum allows for a lower production rate of radioactive waste by radiative capture and for the burn-out of a fraction of the long-lived actinides. However, both the reactor dynamics and the burn-out of fuel and actinides depend on the 
neutron spectrum. Conventionally, the neutron spectrum in a reactor is measured by activation foil analysis. Indeed, the activation of an isotope can be related to the convolution of the neutron flux with the isotope activation cross-section. But this complex, offline, procedure introduces large systematic uncertainties. A simple online technique is necessary for characterization of reactor transients. For this purpose we developed a novel neutron spectrometer based on a ${ }^{6} \mathrm{Li}$ converter sandwiched between two CVD diamond detectors. The energy of the incident neutron converts completely into the energy of charged particles through the ${ }^{6} \mathrm{Li}(\mathrm{n}, \alpha) t$ reaction. This allows for an event-byevent neutron energy measurement with the advantages of a solid state detector. Moreover, the ${ }^{6} \mathrm{Li}(\mathrm{n}, \alpha) t$ reaction is highly exothermic with $Q=4.7 \mathrm{MeV}$, which permits to reduce the background by imposing a high detection threshold. The coincidence between two crystals further suppresses noise and competing reactions.

In this paper we describe the spectrometer assembly and two calibration experiments. The details of detector development are given in Section 2. Measurements of the detector response to thermal neutrons, made at a TRIGA reactor, are discussed in Section 3, while the fast neutron experiment at a DD-fusion source is detailed in Section 4.

\section{Detector construction}

The sandwich spectrometer prototype was built from two SingleCrystal Diamond detectors (SCD), identified as SCD398 and SCD1517, grown at the laboratories of the University of Rome "Tor Vergata".

Each SCD was grown on a $4 \times 4 \times 0.4 \mathrm{~mm}^{3}$ HPHT substrate, and had the same structure, as shown in Fig. 1. These detectors are composed of three main layers: p-type diamond, intrinsic diamond and metal contact. This diode-like structure is described in Refs. [3,4] and allows the readout of signals from the intrinsic diamond layer without removal of the HPHT substrate. The degenerate p-type layer acts as an ohmic contact. Instead, the anode creates a Schottky junction with the underlying intrinsic diamond. In this configuration, the electric current generated by the passage of an ionizing particle in the depletion layer flows across the detector without any barrier. In fact, the Schottky junction at the intrinsic diamond-metal interface accelerates electrons leaving the diamond bulk.

The top contact on the intrinsic diamond side was created by depositing $3 \mathrm{~mm} \times 3 \mathrm{~mm} \times 40 \mathrm{~nm}$ Chromium layer, which was also used as a sticking layer for the two narrow $(0.4 \mathrm{~mm} \times 3 \mathrm{~mm} \times$ $80 \mathrm{~nm}$ ) gold strips shown in Fig. 2. These strips were used to read out the anode signals. On top of the Chromium layer in between the Gold strips a further layer of $3 \mathrm{~mm} \times 2.2 \mathrm{~mm} \times 100 \mathrm{~nm}$ of ${ }^{6} \mathrm{LiF}$ was then deposited on the contact. The LiF compound was chosen as a

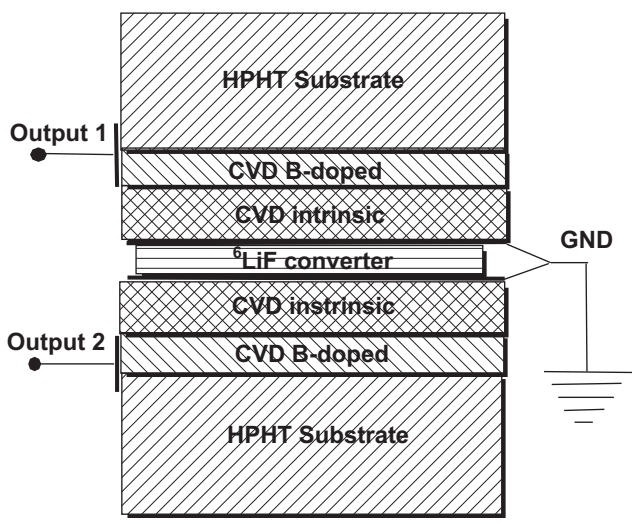

Fig. 1. Design of the sandwich spectrometer with its main components: HPHT diamond substrate, B-doped diamond layer, intrinsic diamond layer, metallic contact and LiF converter.

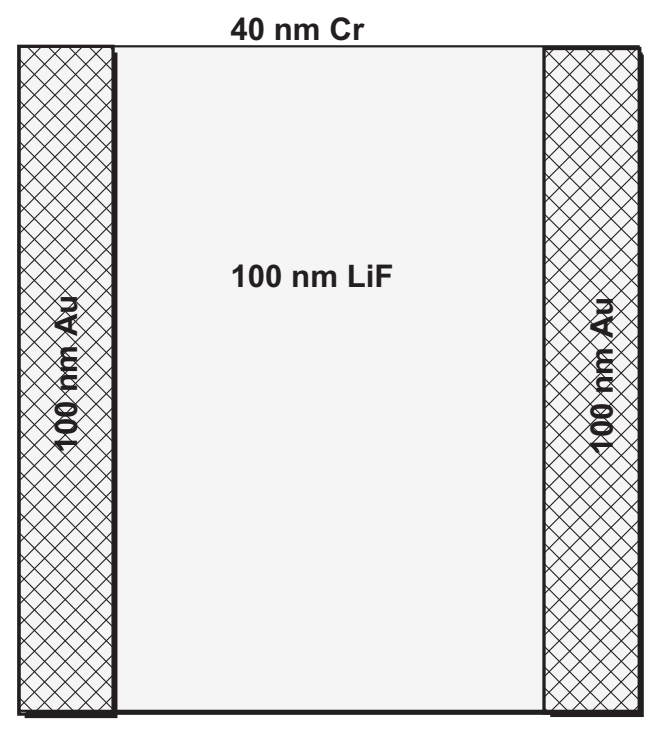

Fig. 2. Gold strips and LiF converter with Chromium contact underneath, deposited on top of the intrinsic CVD diamond crystal.

Table 1

Diamond detector characteristics.

\begin{tabular}{|c|c|c|c|c|}
\hline SCD & $\begin{array}{l}\text { P-doped layer } \\
\text { thick. } \\
{[\mu \mathrm{m}]}\end{array}$ & $\begin{array}{l}\text { Intrinsic layer } \\
\text { thick. } \\
{[\mu \mathrm{m}]}\end{array}$ & $\begin{array}{l}\text { Cr contact } \\
\text { thick. } \\
{[\mathrm{nm}]}\end{array}$ & $\begin{array}{l}\text { LiF layer } \\
\text { thick. } \\
{[\mathrm{nm}]}\end{array}$ \\
\hline SCD398 & 23 & 22 & 40 & 100 \\
\hline SCD1517 & 15 & 49 & 40 & 100 \\
\hline
\end{tabular}

neutron converter because of its chemical neutrality. The selected LiF was enriched with ${ }^{6} \mathrm{Li}$ isotope to $96 \%$. The LiF layer is deposited on the top of the metallic anode in such a way that $\alpha$ and $t$ can easily penetrate into the intrinsic diamond depletion layer with minimal loss of energy. In fact, the assembly leaves only a $50 \mu \mathrm{m}$ gap to accommodate the central ground microwire.

The active volume of each detector consists of its intrinsic diamond layer. The thicknesses of the Boron doped (P-type) and intrinsic diamond layers were different for the two detectors, as shown in Table 1 . The thicknesses of the chromium contacts, and of the ${ }^{6} \mathrm{LiF}$ layers, instead, were the same within the uncertainties of the measurement system of the thermal evaporator .

The "sandwich" structure is realized by means of two small double-layer Printed Circuit Boards (PCB) (35 $\mu \mathrm{m}$ golden copper on $0.8 \mathrm{~mm}$ FR4), to which diamonds are glued. Each PCB has a ground plane on one side and signal traces on the other. The Ptype layers are connected to the signal traces with a droplet of conductive glue. The top electrode, which is shared by the two diamonds, is connected by two microwires running along the Gold strips, not covered by LiF, and soldered to the ground plane through four vias. The wires are made of Copper (diameter $50 \mu \mathrm{m}$ ) plated with $2 \mu \mathrm{m}$ of galvanically deposited gold. Each PCB has four golden-plated connectors which guarantee the integrity of the ground plane and the routing of electrical signals, but also a precise mechanical alignment between the diamonds and the wires when the "sandwich" is closed, i.e. when the two PCBs are joined one to each other. The stability of the electrical contact between the wires and the Gold strips of both diamonds is ensured by a U-shaped spring applies a small force pushing the PCBs one toward the other. The two PCBs have different length, allowing RG62 signal cables to be soldered on dedicated pads on the longer board. 


\section{Measurement at TRIGA reactor}

In order to characterize the detector and to evaluate the absolute amount of ${ }^{6} \mathrm{Li}$ isotope deposited on the sensitive detector area a measurement in a calibrated thermal neutron flux was performed. The measurement was carried out at TRIGA reactor located at Laboratory for Applied Nuclear Physics (LENA) of the Pavia University. The reactor has variable power from few $\mathrm{kW}$ up to $250 \mathrm{~kW}$, accurately monitored by an in-core compensated fission chamber system [5]. It features several irradiation channels, among which a large graphite thermal column. The neutron flux in the column is almost perfectly Maxwellian reaching values of $1.2 \times 10^{10} \mathrm{n} / \mathrm{cm}^{2} / \mathrm{s}$ at $250 \mathrm{~kW}$. The detector was placed in a specific position inside the thermal column, where the neutron flux was carefully determined [6]. The data were acquired at a few values of reactor power ranging from $1 \mathrm{~kW}$ up to $100 \mathrm{~kW}$.

\subsection{Experiment}

The sandwich spectrometer prototype under test was made of two CVD diamonds SCD398, identified as channel 1, and SCD1517, identified as channel 2 . The signals from the two diamonds were connected via $2.5 \mathrm{~m}$ RG62 cables to Silena 205 charge preamplifiers. The amplified signals were driven to the Ortec 673 Spectroscopy amplifiers where they were further amplified and filtered with $2 \mu$ s shaping time. The final signals were acquired by CAEN V785 peak sensing ADC. The trigger signals were taken from CAEN V895 discriminator, which was connected to the secondary preamplifier output, amplified by Ortec 460 delay line amplifiers with 200 ns shaping time.

The Data AcQuisition (DAQ) system was based on VME modular electronics. In particular, Concurrent Tech. VX813-09x single board computer was used as VME controller as well as the acquisition host. The VX813-09x ran 32-bit CentOS 6 Linux operating system with native Tsi148 VME controller drivers. The CAEN V785 peak sensing ADC was configured to generate interrupts on VME bus when the number of events in its multi-event buffer exceeded the imposed threshold. When the interrupt was received by the VX813/09x board, it copied all the data from the V785 buffer through a fast DMA MBLT transfer and sent them together with the interrupt time stamp to a secondary thread. This secondary thread was buffering and saving the data on a fast SATA SSD. The amount of data and the event rate were relatively small, compared to the host memory size, thus VME transfer and disk writing speed were sufficient to run acquisition without additional delays. In order to measure the system dead time, the discriminated signals were counted on a CAEN V830 scaler during runtime.

\subsection{Data analysis}

For the absolute normalization of the measurement two independent data were used. The first was provided by the TRIGA power monitoring system combined with the power-to-flux conversion from Ref. [6]. For the second, an independent fission chamber with known sensitivity of $3 \times 10^{6} \mathrm{n} / \mathrm{cm}^{2} \mathrm{~s} / \mathrm{cps}$ [7] was installed. The fission chamber signal was amplified by an Ortec 142B charge sensitive preamplifier and an Ortec 572A Spectroscopy amplifier and then discriminated by an Ortec 934 discriminator. The discriminator logical output was connected to the CAEN V830 scaler, counting all the signals within DAQ runtime. The measured fission chamber signal rate was found to be in good agreement with the TRIGA power monitoring system. The correlation between the sandwich detector event rate and the fission chamber signal rate was found to be linear within uncertainties on the interval of two orders of magnitude, as shown in Fig. 3. The locations of the fission chamber and the sandwich spectrometer were different leading to a somewhat large $\chi^{2}$ value.

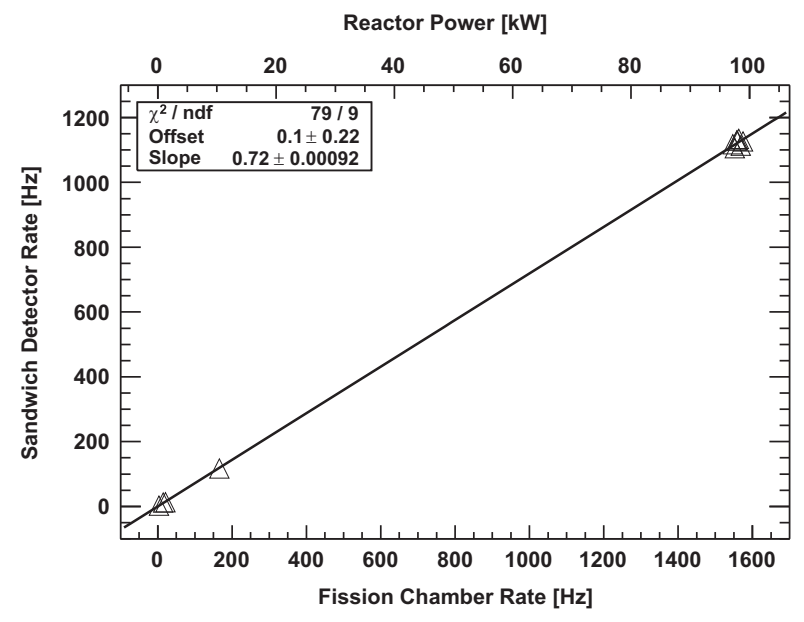

Fig. 3. Correlation between sandwich detector event rate, fission chamber rate and reactor power.

Since the DAQ system was configured to acquire both ADCs when a trigger in any of the two channels occurred (OR configuration), the coincidence events were selected off-line. The selection was based on the application of an off-line threshold to both ADCs, determined as the ADC channel above which the measured yield was more than 100 times higher than a possible tail from the ADC pedestal. The discarded events were mainly those in which one of two reaction products $\alpha$ or $t$ was lost. This occurred when one of reaction products was emitted in the $50 \mu \mathrm{m}$ gap between the two crystals.

The sandwich detector response to the neutron flux was modeled using Geant version 4.9.5.p01. The detector was described in detail, including PCB support with its metallization, HPHT diamond substrate, B-doped CVD diamond cathode substrate, intrinsic CVD diamond, $\mathrm{Cr}$ anode layer, LiF layer, gold strips and gold-plated cooper wires. Similar simulations were performed with MCNP, but with $\mathrm{Cr}$ contacts of $100 \mathrm{~nm}$ which resulted in a larger energy loss of $\alpha$ and a slight shift in its peak position. This difference was not important in efficiency evaluation, but allowed us to quantify the effect of finite contact thickness on the energy resolution. In these simulations the neutron flux of the TRIGA thermal column [6] was generated isotropically on a spherical surface around the spectrometer of area about $1 \mathrm{~cm}^{2}$. Events with energy deposited in any of two crystals of the sandwich sensitive volume above threshold were selected.

The calibration of the energy deposited in each crystal was based on the energy of the produced $t$, corrected for the energy lost in the LiF layer and the $\mathrm{Cr}$ contacts. The ADC pedestal peak position was taken as the zero energy point. The obtained deposited energy distribution in the SCD398 crystal is shown in Fig. 4. The high energy peak, due to the absorption of $t$, has an almost Gaussian shape. The resolution of $t$-peak was found to be $42 \mathrm{keV}$ for SCD398 and $22 \mathrm{keV}$ for SCD1517. These values are only slightly larger than the measured pedestal widths of 38.5 and $18.5 \mathrm{keV}$, respectively. The intrinsic resolution due to the energy loss in LiF layer and $\mathrm{Cr}$ contacts is estimated to be about $5 \mathrm{keV}$.

The lower energy peak in Fig. 4 is due to the absorption of $\alpha$. It exhibits a significant low-energy tail because of the higher energy loss of $\alpha$ s in LiF and $\mathrm{Cr}$.

The sum of the two energies deposited in the two crystals gives the incident neutron energy increased by the reaction $Q$-value. For thermal neutrons the incident energy is much lower than the detector energy resolution and therefore in this case the total energy must be equal to the reaction $Q$-value (4.7 MeV). Summing up the energies of the two crystals indeed leads to an asymmetric peak at around 4.7 MeV, as shown in Fig. 5. The peak resolution was found to be $73 \mathrm{keV}$, compared to the intrinsic resolution of $21 \mathrm{keV}$ due to energy losses in $\mathrm{LiF}$ layer and $\mathrm{Cr}$ contacts. 


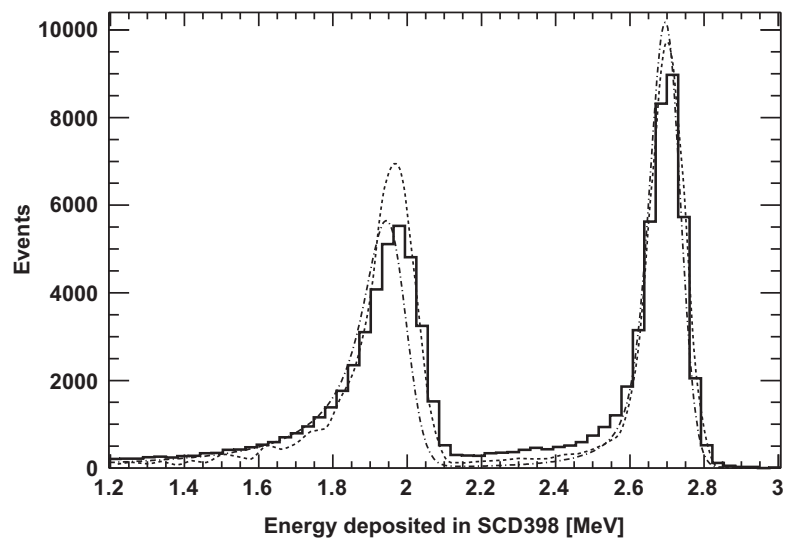

Fig. 4. Energy deposited in the single diamond SCD398 of the spectrometer in comparison with Geant4 (dashed line) and MCNP (dot-dashed line) simulations. In MCNP simulations $100 \mathrm{~nm} \mathrm{Cr}$ contacts were assumed, while in Geant4 they were set to $40 \mathrm{~nm}$.

The main goal of this measurement was the evaluation of the absolute efficiency of the spectrometer in detecting thermal neutrons. The spectrometer inefficiency to the produced $t+\alpha$ pair was fairly low due to the small angular acceptance of the $50 \mu \mathrm{m}$ gap between the crystals and the almost complete coverage of the LiF layer by the underlying $\mathrm{Cr}$ anode. However, the amount of ${ }^{6} \mathrm{Li}$ deposited by evaporation on the diamond $\mathrm{Cr}$ contacts was known with a large uncertainty. To determine it precisely, a comparison between the data and Monte Carlo was used.

In order to normalize the simulations to the same neutron flux seen by the spectrometer the reconstructed Geant 4 distributions were rescaled by the factor

$L_{\text {sim }}=\phi_{n}^{\text {tot }} t_{\text {run }} \frac{S_{g e n}}{N_{g e n}}$

where $\phi_{n}^{\text {tot }}$ is the total neutron flux measured by the fission chamber, $t_{\text {run }}$ is the DAQ runtime, $N_{\text {gen }}$ is the total number of neutrons generated on the surface of area $S_{\text {gen }}$. The thickness of LiF was first assumed to be equal to the nominal value of $200 \mathrm{~nm}$ and then adjusted as a free parameter to reproduce the data. The obtained in this way thickness of LiF was found to be $80 \mathrm{~nm}$. This effective number includes also all other spectrometer inefficiencies not accounted for in the Geant 4 simulation.

In order to compare Geant 4 simulations to the data, an additional Gaussian smearing due to electronic readout noise was added to the reconstructed energies. The obtained simulations describe the data fairly well (see Figs. 4 and 5), in particular in the region of $t$-peak, which has almost a Gaussian shape. Instead the $\alpha$-peak is more asymmetric and features a larger l.h.s. tail due to higher energy loss of $\alpha$ particles in LiF and $\mathrm{Cr}$. The total deposited energy distribution is also well reproduced.

The correlation between the signals in the two crystals, shown in Fig. 6, reveals an important difference: the enhanced tail of the $t$-peak in SCD398. This was related to the crystal depletion layer thickness, in case of SCD398 smaller than the $2.7 \mathrm{MeV} t$ range in the diamond. In fact, those $t$ incident on SCD398 almost perpendicular to its surface were not completely absorbed. This behavior was reproduced by Geant4 simulations assuming a depletion layer thickness of $18 \mu \mathrm{m}$.

The fast charge amplifier developed by Cardarelli [8] was also used for readout together with SIS3305 $5 \mathrm{Gs} / \mathrm{s}$ digitizer. It showed results consistent with the standard charge sensitive electronics, but with drastically reduced dead time. However, the energy resolution was found to be at least two times worse.

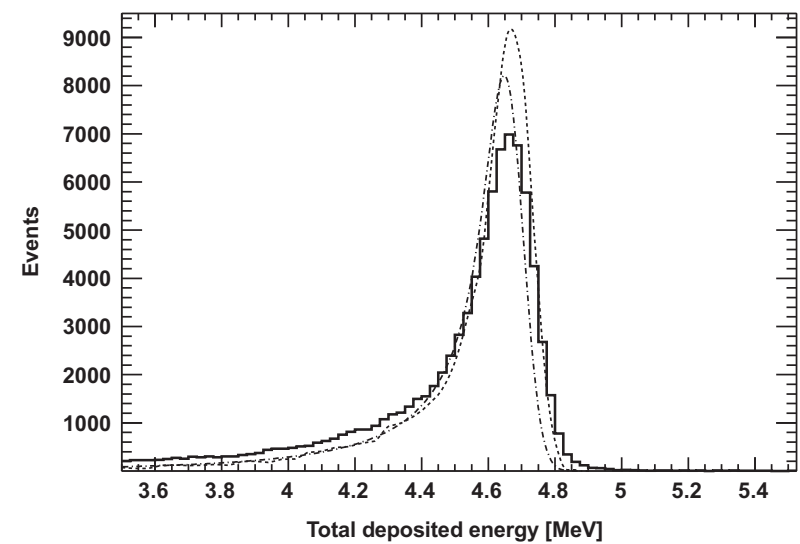

Fig. 5. The same as in Fig. 4, but for the total energy deposited in the spectrometer.

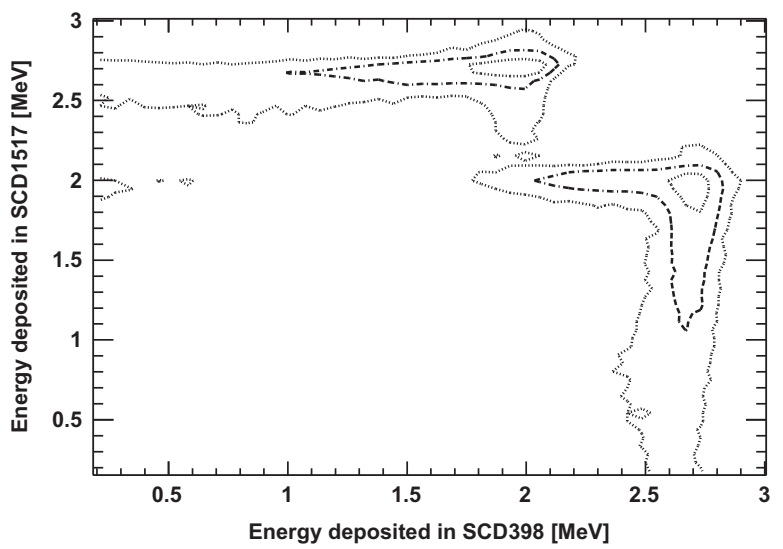

Fig. 6. Correlation between deposited energy spectra in the two diamonds of the spectrometer. $t$-peak at $2.7 \mathrm{MeV}$ in one crystal corresponds to $\alpha$ peak at $2 \mathrm{MeV}$ in the other. The long tail of the $t$-peak in SCD398 is due to incomplete energy deposition related to the small thickness of SCD398 depletion layer.

\section{Measurement at FNG}

The response of the spectrometer to fast neutrons was measured at the FNG facility of ENEA [9]. The FNG is a neutron generator based on the $\mathrm{T}(\mathrm{d}, \mathrm{n}) \alpha$ and $\mathrm{D}(\mathrm{d}, \mathrm{n}) t$ fusion reactions to produce a nearly isotropic source of $5 \times 10^{10} 14-15 \mathrm{MeV} \mathrm{n} / \mathrm{s}$ or $5 \times 10^{8} \mathrm{n} / \mathrm{s} 2.5-3 \mathrm{MeV} \mathrm{n} / \mathrm{s}$, respectively. The neutron source strength is continuously monitored by counting the recoiling $\alpha$ or $t$ particles associated with each neutron produced by the DT or DD reaction. The recoiling charged particles are counted by means of a small silicon surface barrier detector incorporated in the beamline. The resulting uncertainty on the source strength measurement is lower than $4 \%$. The FNG target is installed at $4 \mathrm{~m}$ above the floor and $4 \mathrm{~m}$ from walls and ceiling thus reducing the contribution from neutron rescattering on environmental materials.

\subsection{Experiment}

The detector was installed at $2.5 \mathrm{~cm}$ in front of the TiD target (zero degrees with respect to $D$ beam). During the experiment FNG was operated in DD-mode with a beam current of $1 \mathrm{~mA}$ and a total 2.5$3 \mathrm{MeV}$ neutron yield of $5.7 \times 10^{8} \mathrm{n} / \mathrm{s}$. Some contamination of tritium in the target and beamline generated a background of $14-15 \mathrm{MeV}$ DT neutrons. The total neutron yield was continuously monitored by the recoil detector, whose rate variations during the experiment are shown in Fig. 7. The sandwich spectrometer rate, measured in four large runs, followed recoil detector rate. Moreover, to better determine the flux of fast neutrons at the spectrometer location, two ${ }^{115}$ In foils 
were installed in front and at the back of the spectrometer. In order to calibrate the ADC energy scale a polyethylene moderator was installed behind the detector.

DAQ was run in coincidence (AND) configuration at the total rate of about $60 \mathrm{~Hz}$. At such a low event rate the DAQ dead time was negligible.

\subsection{Data analysis}

The irradiated ${ }^{115}$ In $95.7 \%$ NA foils were measured by a $60 \%$ relative efficiency HPGe detector immediately after the experiment and the strengths of a few $\gamma$ emission lines of two reactions were determined. The first reaction was ${ }^{115} \mathrm{In}\left(\mathrm{n}, \mathrm{n}^{\prime}\right)^{115 \mathrm{~m}} \mathrm{In}$, where the produced excited state decays with 4.49 h half-life by emitting a $336 \mathrm{keV} \gamma$ with $45.8 \%$ probability. This reaction is mostly sensitive to the $2.5-3 \mathrm{MeV}$ neutrons from DD-fusion. The second reaction measured by the HPGe was ${ }^{115}$ In $(\mathrm{n}, \gamma)^{116 \mathrm{~m}} \mathrm{In}$ where the produced metastable nucleus decays with 54.15 min half-life by emitting a number of $\gamma \mathrm{s}$ with various probabilities: $417 \mathrm{keV}$ (29.2\%), $819 \mathrm{keV}$ (11.5\%), $1097 \mathrm{keV}$ (56.2\%), $1294 \mathrm{keV}$ (84.4\%), $1507 \mathrm{keV}$ (10\%). The two measured activities together with a detailed input neutron spectrum calculated by MCNP simulations were used by SAND II [10] unfolding procedure to obtain the absolute neutron flux as a function of neutron energy. The MCNP simulations describe in detail the FNG neutron source and were carefully tested in Ref. [11].

Because of the very low signal rate in this measurement the ADC spectra were dominated by accidental coincidences. These were mostly events due to electronic noise and the elastic neutron scattering on carbon nuclei. The events in which both ADCs were above the channel corresponding to more than a 100 times the pedestal tail were selected as coincidences.

The ADC energy calibration was accomplished by using the position of the thermal neutron induced $t$-peak, clearly visible in both diamonds. The zero energy scale was obtained from the pedestal peak position.

Preliminary simulations showed that most of the $3 \mathrm{MeV}$ neutron events deposit more than $1.4 \mathrm{MeV}$ in each crystal. Hence, the off-line threshold of $1.4 \mathrm{MeV}$ was applied in order to remove the elastic scattering contribution and other accidental coincidences.

Once the accidental coincidences were removed, the energies deposited in the two crystals were summed up to obtain the total deposited energy showed in Fig. 8. The obtained distribution exhibits a large peak at $4.7 \mathrm{MeV}$ due to thermal neutron detection as well as lower peak at $7.7 \mathrm{MeV}$ due to direct $3 \mathrm{MeV}$ neutrons from DD source.

Comparisons of data to Geant 4 simulations performed with a realistic neutron spectrum revealed the complex structure of the

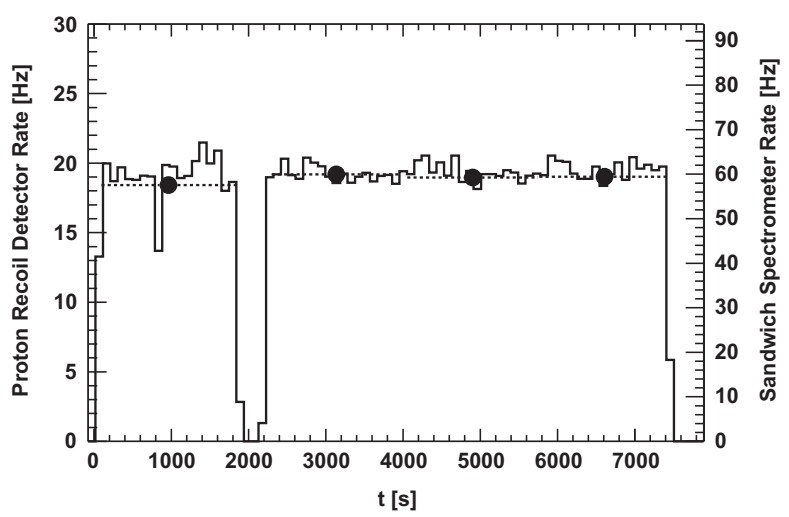

Fig. 7. Rate of the FNG recoil detector (histogram) in comparison to the sandwich spectrometer rate (full circles, dashed lines show run duration) rescaled for comparison.

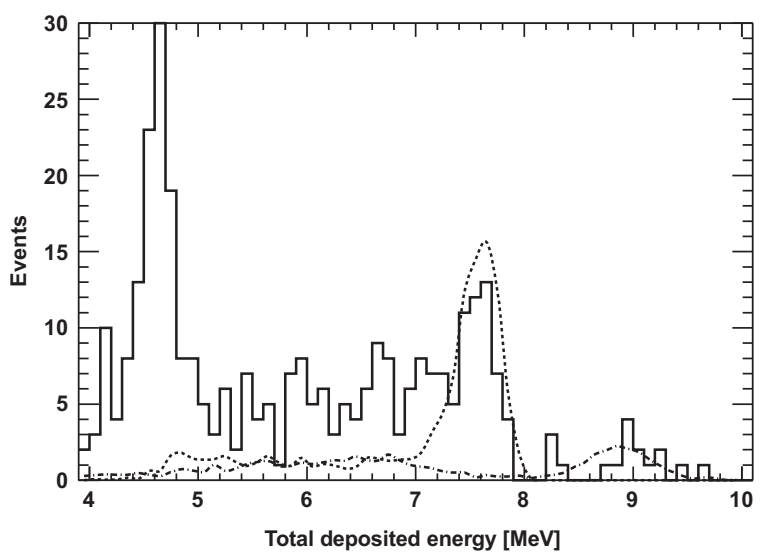

Fig. 8. Total energy deposited in the spectrometer in comparison with Geant4 simulations of DD neutrons (dashed line) and DT neutrons (dot-dashed line). The peak at $4.7 \mathrm{MeV}$ is due to thermalized neutrons used for energy calibrations, the tail at the left of $7.7 \mathrm{MeV}$ peak (3 MeV neutrons) is partially due to DT neutron inelastic reactions in the diamond and due to the tail of scattered $3 \mathrm{MeV}$ neutrons.

measured distribution. Indeed, few events seen at $9 \mathrm{MeV}$ were due to direct $15 \mathrm{MeV}$ neutron conversion into ${ }^{9} \mathrm{Be}+\alpha$, where $\alpha$ produced in the first diamond traveled into the second crystal to generate the coincidence.

The l.h.s. shoulder of the $7.7 \mathrm{MeV}$ peak is made of two contributions of similar magnitude: carbon disintegration into $3 \alpha$ s by incident $15 \mathrm{MeV}$ neutrons and the tail of DD peak from the initial neutron spectrum. The latter tail was produced by the neutron rescattering from surrounding materials.

The resolution of the detector in this experiment was slightly worse than in the TRIGA measurement as one can see from the thermal peak $\sigma$ of $\sim 90 \mathrm{keV}$. This was related to a higher level of environmental electronic noise in the experimental hall.

Prototype II featuring $5 \mathrm{~m}$ RG62 connection cable was installed at $90^{\circ}$ with respect to $D$ beamline at $3 \mathrm{~cm}$ distance from the target center. The same analysis was performed on its data. The obtained results (see Fig. 9) are in good agreement with Geant4 simulations. The expected neutron spectrum features a peak at $2.45 \mathrm{MeV}$ with twice smaller width ( $77 \mathrm{keV}$ instead of $177 \mathrm{keV}$ at $0^{\circ}$ ). In the measured data the peak widths are enhanced by the spectrometer energy resolution, but the ratio between 90 and $0^{\circ}$ widths is similar: $114 \mathrm{keV}$ and $204 \mathrm{keV}$, respectively.

\section{Backgrounds}

Thanks to highly exothermic reaction on ${ }^{6} \mathrm{Li}$, backgrounds can be neglected when the maximum neutron energy (with significant flux) remains below $6 \mathrm{MeV}$. In fact, the most probable reaction, neutron elastic scattering off carbon nuclei, deposits only $<28 \%$ of the neutron energy in the detector. Therefore it can be easily removed by a high threshold ( $1.4 \mathrm{MeV}$ for neutron up to $6 \mathrm{MeV}$ ) with minimal efficiency loss. All the inelastic reactions on carbon, except for unlikely radiative capture and excitation of the first ${ }^{12} \mathrm{C}$ level, occur above $6 \mathrm{MeV}$.

The spectrometer has a low sensitivity to $\gamma$-rays, thanks to its low $Z=6$ value. Moreover, due to the small thickness of the crystals $(50 \mu \mathrm{m})$ a significant energy loss by generated electrons in the spectrometer sensitive volume is very unlikely.

Therefore, we neglected all backgrounds except for contributions from other parts of the neutron spectrum. These other parts of the neutron spectrum seen in FNG experiment are mostly due to $\mathrm{T}$ contamination in the TiD target, yielding $15 \mathrm{MeV}$ neutrons, and due to the tail of $3 \mathrm{MeV}$ neutrons scattered from surrounding materials. 


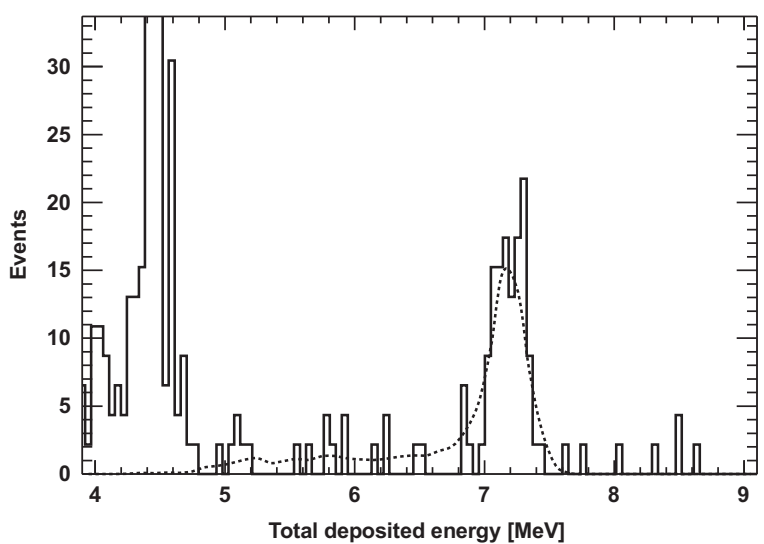

Fig. 9. The same as if Fig. 8 but measured at $90^{\circ}$ with respect to deuteron beam with Prototype II. Peak width is almost factor of two smaller than at $0^{\circ}$.

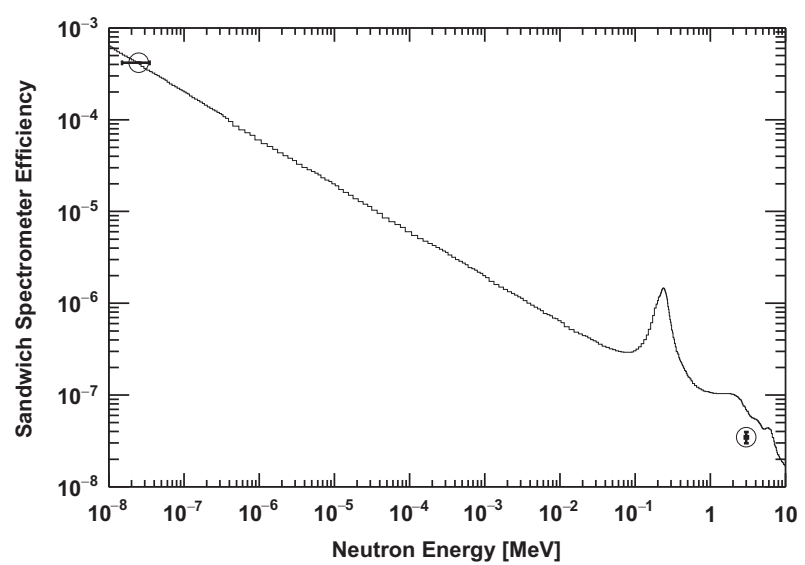

Fig. 10. Neutron detection efficiency of sandwich spectrometer for the neutrons incident on $0.066 \mathrm{~cm}^{2}$ active surface in comparison with ${ }^{6} \mathrm{Li}(\mathrm{n}, \mathrm{t}) \alpha$ cross-section rescaled by surface ${ }^{6} \mathrm{Li}$ atom density.

\section{Systematic uncertainties}

The low statistics of FNG measurements allowed only a $10 \%$ statistical precision. Thus, we considered only major contributions which were of the order of these uncertainties.

The largest uncertainties come from the normalization of the neutron flux. In TRIGA measurement the fission chamber sensitivity of $3 \times 10^{6} \mathrm{n} / \mathrm{cm}^{2} \mathrm{~s} / \mathrm{cps}$ [7] was established with uncertainty of $8 \%$, while the neutron flux in the TRIGA thermal column as a function of reactor power had $2.5 \%$ precision.

In FNG measurement we relied on the ${ }^{115}$ In activation foil analysis which had an overall uncertainty of $20 \%$. This value combines both uncertainties due to the position mismatch and due to the spectrum reconstruction from the excited level activity measurement.

\section{Results}

The detection efficiency of the sandwich spectrometer prototype for neutrons incident on its ${ }^{6} \mathrm{LiF}$ layer area of $0.066 \mathrm{~cm}^{2}$ was measured at two neutron energies: thermal (25 meV) and $3 \mathrm{MeV}$. The obtained energy dependence was found to be in good agreement with the known ${ }^{6} \mathrm{Li}(\mathrm{n}, \alpha) t$ cross-section rescaled by the surface atom density as shown in Fig. 10 and in Table 2. At $3 \mathrm{MeV}$ the measured efficiency was 30\% lower than the crosssection, but well described by Geant 4 simulations. This is related
Table 2

Measured efficiency of the neutron spectrometer for the neutrons incident on $0.066 \mathrm{~cm}^{2}$ active surface.

\begin{tabular}{ll}
\hline$E_{n}$ & Efficiency \\
\hline $25 \mathrm{meV}$ & $4.2 \times 10^{-4} \pm 0.00003 \% \%_{\text {stat. }} \pm 8 \%_{\text {sys. }}$ \\
$3 \mathrm{MeV}$ & $3.5 \times 10^{-8} \pm 14 \%_{\text {stat. }} \pm 20 \%{ }_{\text {sys. }}$ \\
\hline
\end{tabular}

to a broader energy distribution of reaction products, some of which carry energy below the selected threshold.

In order to obtain detector sensitivity in $\mathrm{cps} /\left(\mathrm{n} / \mathrm{cm}^{2} \mathrm{~s}\right)$ the efficiency has to be multiplied by the spectrometer active surface area of $0.066 \mathrm{~cm}^{2}$.

\section{Conclusions}

A neutron spectrometer for fast nuclear reactors based on ${ }^{6} \mathrm{LiF}$ converter sandwiched between two CVD diamond detectors was developed. This work was mostly dedicated to the measurements of the spectrometer efficiency as a function of neutron energy. Because of small efficiency values only high neutron flux facilities could be used for this purpose. The first prototype was assembled and calibrated with thermal neutrons at TRIGA reactor of LENA and with $3 \mathrm{MeV}$ neutrons at FNG facility of ENEA. The energy dependence of the efficiency of the spectrometer was found to be in good agreement with expectations based on Geant4 and MCNP simulations. The neutron energy resolution was found to be as low as $73 \mathrm{keV}$ (RMS). The most important contribution in the resolution function was given by electronics noise, while the intrinsic resolution of the spectrometer was estimated to be $21 \mathrm{keV}$. The latter derives from the stochastic energy loss in LiF converter and Cr contacts.

Due to its low efficiency the spectrometer is suited for neutron fluxes $>10^{7} \mathrm{n} / \mathrm{cm}^{2}$ s. Moreover, because of the higher sensitivity to thermal neutrons and the opening of inelastic reaction channels on ${ }^{12} \mathrm{C}$ above $6 \mathrm{MeV}$ the most appropriate usage of the spectrometer is the measurement of fission spectrum. Such a neutron spectrum can be found in fast fission reactors or spent fuel elements.

\section{Acknowledgments}

The authors would like to acknowledge the excellent support provided during the experiments by the staff and technical services of LENA and FNG facilities. We thank Prof. Saverio Altieri and collaborators of Pavia University for the help with fission chamber measurements in TRIGA reactor. This work was supported by the Istituto Nazionale di Fisica Nucleare INFN-E project.

\section{References}

[1] W. de Boer, et al., Physica Status Solidi 204 (2007) 3009.

[2] M. Bruzzi, H. Sadrozinski, A. Seiden, Nuclear Instruments \& Methods A 579 (2007) 754.

[3] M. Angelone, et al., IEEE Transactions on Nuclear Science NS-56 (2009) 2275. [4] S. Almaviva, et al., Applied Radiation and Isotopes 67 (2009) 183.

[5] A. Borio di Tigliole, et al., Home-made refurbishment of the instrumentation and control system of the triga reactor of the university of pavia (2008). Proceedings of the 4. World TRIGA Users Conference, Lyon (France); 7-10 Sep 2008, INIS and NKM - Database Production and Imaging Group, Vienna (Austria), p. 142-170, http://www.iaea.org/inis/collection/NCLCollection Store/_Public/40/007/40007412.pdf.

[6] N. Protti, S. Bortolussi, M. Prata, P. Bruschi, S. Altieri, D. Nigg, Transactions of the American Nuclear Society 107 (2012) 1269. 
[7] A. Altieri, Private Communication.

[8] R. Cardarelli, A.D. Ciaccio, L. Paolozzi, Nuclear Instruments \& Methods A 745 (2014) 82.

[9] M. Martone, M. Angelone, M. Pillon, Journal of Nuclear Materials B 212 (1994) 1661.
[10] W. McElroy, S. Berg, T. Crockett, R. Hawkins, A Computer-automated 407 Iterative Method for Neutron Flux Spectra Determination, Report AFWL-TR 6741, 1967.

[11] M. Angelone, M. Pillon, P. Batistoni, M. Martini, M. Martone, V. Rado, Review of Scientific Instruments 67 (1996) 2189. 